\title{
ON A CONJECTURE ON SIMPLE GROUPS
}

\section{N. HERSTEIN}

The purpose of this paper is to rephrase a conjecture about simple groups into the language of linear algebra.

Let $G$ be a group of finite order $o(G)$. Then by $\Gamma_{p}$ we shall mean the group ring of $G$ over a field of characteristic $p$ (for instance the integers modulo $p$ ). We shall denote the radical of $\Gamma_{p}$ by $N_{p}$. If $p=0$ or $p \nmid o(G)$, then it is known that $N_{p}=(0)$; and if $p \mid o(G), N_{p} \neq(0)$.

We now consider the following two assertions:

(A) If $G$ is a simple group of odd order, o $(G)$ is a prime.

(B) If $G$ is a group of odd order $o(G)$, then for some prime $p, p \mid o(G)$, we can find a $g \in G, g \neq 1$, such that $g-1 \in N_{p}$.

The theorem which we propose to prove is:

Theorem. (A) is equivalent to (B).

1. (B) implies (A).

Definition. $U_{p}=\left\{g \in G \mid g-1 \in N_{p}\right\}$.

LEMMA 1. $U_{p}$ is a normal p-subgroup of $G$.

Proof. (a) $U_{p}$ is a subgroup of $G$, for if $g_{1}, g_{2} \in U_{p}$, then since $N_{p}$ is a left-ideal of $\Gamma_{p}, g_{1}\left(g_{2}-1\right)+\left(g_{1}-1\right)=g_{1} g_{2}-1 \in N_{p}$.

(b) $U_{p}$ is normal, for if $g-1 \in N_{p}$, since $N_{p}$ is a two-sided ideal of $\Gamma_{p}, h(g-1) h^{-1}=h g h^{-1}-1 \in N_{p}$ for all $h \in G$.

(c) If $g-1 \in N_{p}$, then for some integer $S,(g-1)^{p^{*}}=0=g^{p^{*}}-1$. So if $g \in U_{p}, g$ is of order $p^{8}$ for some $s$. So $U_{p}$ is a $p$-group.

Corollary. (B) implies (A).

Proof. By (B), $U_{p} \neq 1$ for some $p \mid o(G)$. Hence since $G$ is simple, and since $U_{p}$ is a normal subgroup of $G, U_{p}=G$. Thus $G$ is of order $p^{*}$, and $G$ being simple, $s=1$. Hence (B) implies (A).

2. (A) implies (B).

LEMMA 2. (A) implies that every group of odd order is solvable.

Proof. Let $G=G_{1} \supset G_{2} \supset \cdots \supset G_{r}=1$ be a composition series for $G$. Since the $G_{i} / G_{i+1}$ are simple and of odd order, by (A) they must be of prime order; hence the lemma is proved.

Since a solvable group contains a normal $p$-subgroup $[1$, p. 25 , 1949.

Presented to the Society, December 29, 1949; received by the editors May 5, 
Theorem 20], ${ }^{1}$ we immediately obtain, using Lemma 2 , the following lemma.

Lemma 3. (A) implies that if $G$ is of odd order, then it contains a normal p-subgroup.

For groups of certain orders the $N_{p}$ can be completely described. This is true for $p$-groups. If $G$ is of order $p^{8}$, then for every $g \in G$, $g-1 \in N_{p}[2$, p. 176 , Theorem 1.2 or 3, p. 239]. For our case it is sufficient to use the weaker result:

Lemma 4. If $G$ is of order $p^{*}$, then for some $g \neq 1$ in $G, g-1 \in N_{p}$.

Proof. Since $G$ is of order $p^{*}$, it has a nontrivial center $C$. Let $g \neq 1$ be in $C$. Then since $g-1$ is in the center of $\Gamma_{p}$, and since $(g-1)^{p^{*}}$ $=g^{p^{*}}-1=1-1=0, g-1 \in N_{p}$.

Suppose that $S$ is the normal $p$-subgroup of Lemma 3. An element of the form $g-1 \in \Gamma_{p}$ is in $N_{p}$ if and only if for every irreducible representation $\phi$ of $G, \phi(g)=1$. Clifford's theorem [4, p. 534, Theorem 1] reduces the irreducible representations of $G$ to irreducible representations (or into ones fully reducible into irreducible components) of $S$. For the $g$ of Lemma 4 in $S$, for every irreducible representation $\phi$ of $S, \phi(g)=1$. So by Clifford's theorem, for every irreducible representation $\phi$ of $G, \phi(g)=1$. Thus $g-1 \in N_{p}$. And so we have shown the following lemma.

Lemma 5. (A) implies (B).

\section{BIBLIOGRAPHY}

1. A. Speiser, Gruppen der endlichen Ordnung.

2. S. A. Jennings, The group ring of a p-group over a modular field, Trans. Amer. Math. Soc. vol. 50 (1941).

3. Lombardo-Radice, Intorno alle algebre legato ai gruppi di ordine finito, Rendiconti del Seminario Matematico della università di Roma vol. 3 (1939).

4. A. H. Clifford, Representations induced in an invariant subgroup, Ann. of Math. vol. 38 (1937).

UNIVERSITY OF KANSAS

\footnotetext{
1 Numbers in brackets refer to the bibliography at the end of the paper.
} 